\title{
Inhibition of spinal astrocytic c-Jun N-terminal kinase (JNK) activation correlates with the analgesic effects of ketamine in neuropathic pain
}

\author{
Xiao-Peng Mei ${ }^{1,2 \dagger}$, Hui Zhang ${ }^{1 \dagger}$, Wei Wang ${ }^{2}$, Yan-Yan Wei ${ }^{2}$, Ming-Zhu Zhai ${ }^{2}$, Wen Wang ${ }^{2 *}$, Li-Xian Xu ${ }^{1 *}$,
} Yun-Qing $\mathrm{Li}^{\mathrm{i}^{*}}$

\begin{abstract}
Background: We have previously reported that inhibition of astrocytic activation contributes to the analgesic effects of intrathecal ketamine on spinal nerve ligation (SNL)-induced neuropathic pain. However, the underlying mechanisms are still unclear. c-Jun N-terminal kinase (JNK), a member of mitogen-activated protein kinase (MAPK) family, has been reported to be critical for spinal astrocytic activation and neuropathic pain development after SNL. Ketamine can decrease lipopolysaccharide (LPS)-induced phosphorylated JNK (pJNK) expression and could thus exert its anti-inflammatory effect. We hypothesized that inhibition of astrocytic JNK activation might be involved in the suppressive effect of ketamine on SNL-induced spinal astrocytic activation.

Methods: Immunofluorescence histochemical staining was used to detect SNL-induced spinal pJNK expression and localization. The effects of ketamine on SNL-induced mechanical allodynia were confirmed by behavioral testing. Immunofluorescence histochemistry and Western blot were used to quantify the SNL-induced spinal pJNK expression after ketamine administration.
\end{abstract}

Results: The present study showed that SNL induced ipsilateral pJNK up-regulation in astrocytes but not microglia or neurons within the spinal dorsal horn. Intrathecal ketamine relieved SNL-induced mechanical allodynia without interfering with motor performance. Additionally, intrathecal administration of ketamine attenuated SNL-induced spinal astrocytic JNK activation in a dose-dependent manner, but not JNK protein expression.

Conclusions: The present results suggest that inhibition of JNK activation may be involved in the suppressive effects of ketamine on SNL-induced spinal astrocyte activation. Therefore, inhibition of spinal JNK activation may be involved in the analgesic effects of ketamine on SNL-induced neuropathic pain.

\section{Background}

Spinal glial activation is both required and sufficient for neuropathic pain after nerve injury [1-4]. Previous studies have also shown that spinal astrocytes and microglia are two key players in the induction and maintenance of neuropathic pain [5-7]. Specifically, astrocytes play a pivotal role in the maintenance of

\footnotetext{
* Correspondence: wangwen@fmmu.edu.cn; kqmzk@126.com; deptanat@fmmu.edu.cn

+ Contributed equally

'Department of Anesthesiology, School of Stomatology, Fourth Military Medical University, Xi'an, 710032, PR China

${ }^{2}$ Department of Anatomy, Histology and Embryology, K. K. Leung Brain Research Centre, Fourth Military Medical University, Xi'an, 710032, PR China Full list of author information is available at the end of the article
}

spinal nerve ligation (SNL)-induced neuropathic pain [8-10]. Accumulating evidence shows that mitogenactivated protein kinases (MAPKs), activated in spinal glia, play an important role in signaling cascades of inflammatory mediators during nerve injury-induced neuropathic pain [10-14]. There are three major MAPK family members: extracellular signal-regulated kinase (ERK), p38, and c-Jun N-terminal kinase (JNK) [11]. Ligation of the $5^{\text {th }}$ lumbar nerve results in activation of all three MAPKs in spinal cord and dorsal root ganglion (DRG), but each one has its own expression time window and different cellular localization $[8,10,15]$. Activated p38 is specifically localized within microglia but not neurons or the astrocytes in the spinal dorsal horn

\section{Biomed Central}


early after the lesion, which suggests that p38 activation in spinal microglia is likely to have a substantial role in the early phase of neuropathic pain $[15,16]$. Compared with p38, the JNK pathway is specific for spinal astrocyte activation in SNL-induced neuropathic pain $[8,17]$. SNL-induced transient activation of JNK in DRG neurons is involved in inducing neuropathic pain, whereas persistent activation of JNK in spinal astrocytes seems to be critical for maintaining SNL-induced neuropathic pain [8]. In particular, inhibition of JNK activation could alleviate neuropathic pain after SNL $[8,17]$. Therefore, inhibition of the JNK pathway during activation of spinal astrocytes could be the underlying mechanism of action for analgesics.

Intrathecal ketamine has been reported to have an evident analgesic effect on neuropathic pain induced by nerve injury or other causes in animal and clinical studies [18-20]. A number of studies have been carried out to elucidate the mechanisms underlying this analgesic effect, but without satisfying output. Previous study [21] showed that both intraperitoneal and intrathecal ketamine inhibit SNL-induced allodynia; however, intrathecal but not intraperitoneal ketamine suppresses SNL-induced astrocytic activation as reflected by downregulated glial fibrillary acidic protein (GFAP) expression. These results suggest that intraperitoneal ketamine could alleviate SNL-induced neuropathic pain via classical "neuronal-based" mechanisms but, in addition, "astrocyte-related" mechanisms may also be important in underlying the anti-allodynic effect of intrathecal ketamine. Interestingly, ketamine can inhibit lipopolysaccharide (LPS)-induced astrocyte activation [22], and can also decrease LPS-induced JNK phosphorylation (pJNK) to inhibit pro-inflammatory gene expression in macrophages [23]. Therefore, we hypothesized that inhibiting activation of JNK pathway might be involved in the suppressive effect of ketamine on SNL-induced spinal astrocyte activation.

In the present study, the expression and localization of SNL-induced spinal pJNK was detected by immunofluorescence. Then, the effects of ketamine on SNL- induced mechanical allodynia were confirmed by behavioral testing. Finally, changes in SNL-induced pJNK expression after ketamine administration were quantified using immunofluorescence histochemistry and western blot.

\section{Methods}

\section{Animal preparation}

Male Sprague-Dawley rats (180-200 g) were housed in plastic cages, and maintained on a $12: 12 \mathrm{~h}$ light/dark cycle under conditions of $22-25^{\circ} \mathrm{C}$ ambient temperature with food and water available. All efforts were made to minimize animal suffering and to reduce the number of animals used. All experimental procedures received prior approval from the Animal Use and Care Committee for Research and Education of the Fourth Military Medical University (Xi'an, China), and the ethical guidelines to investigate experimental pain in conscious animals [24].

\section{Intrathecal implantation}

Intrathecal implantation was performed by inserting a polyethylene (PE) tube (Becton Dickinson and Company, USA) directly into the subarachnoid space of the lumbar enlargement to inject drugs. Briefly, a midline incision $(3 \mathrm{~cm})$ was made at the back of the rat from the level of the $3^{\text {th }}$ thoracic vertebrae to the lower back, under pentobarbital anesthesia (45 $\mathrm{mg} \mathrm{kg}^{-1}$, i.p.). A pre-measured length of PE-10 tube (I.D. $0.28 \mathrm{~mm}$ and O.D. $0.61 \mathrm{~mm}$ ) was passed caudally from the T8 to the L3 level of the spinal cord, and $2 \mathrm{~cm}$ of the free ending was left exposed in the upper thoracic region. Only animals judged to be neurologically normal and that showed complete paralysis of the tail and bilateral hind legs after administration of $2 \%$ lidocaine $(10 \mu \mathrm{l})$ through the intrathecal catheter were used for the following experiments. Rats were allowed to recover for a 3-5 d period before further use.

\section{Spinal nerve ligation}

To create the rat SNL model, under pentobarbital anesthesia (45 $\mathrm{mg} \mathrm{kg}^{-1}$, i.p.), the left transverse process of the L6 vertebra was first removed to expose the L4 and L5 spinal nerves. The L5 spinal nerve was then carefully isolated and tightly ligated with 6-0 silk thread [25]. The surgical procedure for the sham group was identical to that of the SNL group, except that the spinal nerve was not ligated. The animals were followed for $1 \mathrm{w}$ before intrathecal drug administrations were administered from post operative day (POD) 8 to POD 10 .

\section{Intrathecal drug administrations}

$\mathrm{S}(+)$-ketamine hydrochloride (Sigma, St. Louis, MO, USA) was dissolved and diluted with preservative-free normal saline solution for administration. Normal saline $(0.9 \%)$ was used as the negative control. Animals were divided into 4 groups for administration: a Sham-Saline group ( $\mathrm{n}=10$, a volume of $10 \mu \mathrm{l}$ normal saline was injected into Sham rats), an SNL-Saline group ( $\mathrm{n}=10$, a volume of $10 \mu \mathrm{l}$ normal saline was injected into SNL rats), an SNL-ketamine group ( $\mathrm{n}=30 ; 10$ for each of the 3 subgroups; $10 \mu \mathrm{l}$ of 30,100 or $300 \mu \mathrm{g} \mathrm{kg}{ }^{-1}$ ketamine was injected into SNL rats, respectively); and a Sham-ketamine group ( $\mathrm{n}=30 ; 10$ for each of the 3 subgroups; $10 \mu \mathrm{l}$ of 30,100 or $300 \mu \mathrm{g} \mathrm{kg}^{-1}$ ketamine was injected into Sham rats, respectively). Drugs and normal saline were injected intrathecally over $30 \mathrm{~s}$, followed by a $10 \mu \mathrm{l}$ flush of normal saline. The dosages of ketamine 
used in the present study were chosen based on previous research $[18,21]$ and pilot experiments.

\section{Nociceptive behavioral testing}

Animals were habituated to the testing environment for $3 \mathrm{~d}$ before baseline testing, and then were placed under inverted plastic boxes $\left(30 \times 30 \times 50 \mathrm{~cm}^{3}\right)$ on an elevated mesh floor and allowed to habituate for $30 \mathrm{~min}$ before the threshold testing. Briefly, a logarithmic series of 8 calibrated Semmes-Weinstein monofilaments (von-Frey hairs; Stoelting, Kiel, WI, USA) were applied to the ipsilateral hindpaws to determine the stimulus intensity threshold stiffness required to elicit a paw withdrawal response. Log stiffness of the hairs is determined by $\log 10$ (milligrams $\times$ 10) [4]. The 8 filaments had the following log-stiffness values (value in grams is given in parentheses): 4.17 (1479 mg), 4.31 (2041 mg), 4.56 (3630 mg), 4.74 (5495 mg), 4.93 (8511 mg), 5.07 (11749 mg), 5.18 (15136 mg), and 5.46 $(28840 \mathrm{mg})$. The range of monofilaments (1.479-28.840 gm) produced a logarithmically graded slope when interpolating a $50 \%$ response threshold of stimulus intensity (expressed as $\log 10($ milligrams $\times 10))$ [26]. The behavioral responses were used to calculate the $50 \%$ paw-withdrawal threshold (PWT, absolute threshold), by fitting a Gaussian integral psychometric function using a maximumlikelihood fitting method, as described in detail previously [4]. This fitting method allowed parametric statistical analysis. Assessments were made before surgery for baseline value. Then, behavioral tests were performed once a day until POD 10. Intrathecal drug administrations were carried out $30 \mathrm{~min}$ before behavioral test from POD 8 to POD 10. All the PWT tests were performed in a doubleblind manner.

\section{Rotarod testing}

In order to assess whether the drugs used in the present experiment could influence motor function, which might influence the behavioral results, we performed rotarod tests on drug-administered but operation- and behavioral observation-free rats. Rats with no previous exposure to the rotarod test were placed on the Ugo Basile 7650 Rotarod accelerator treadmill (Ugo Basile, Varese, Italy) set at the minimal speed for training sessions of 1-2 min at intervals of 30-60 min. After this learning period, the animals were placed on to the rotarod at a constant speed of 25 RPM. As the animal took a grip on the drum, the accelerator mode was selected on the treadmill, i.e. the rotation rate of the drum was increased linearly at 20 RPM. Thereafter, the time was measured from the start of the acceleration period until the rat fell off the drum. The cut-off time was $30 \mathrm{~s}$. The test was done $30 \mathrm{~min}$ after intrathecal ketamine or saline once a day for 3 days. The time that the animal remained on the rotarod was recorded and expressed as a percentage of its own baseline value.

\section{Immunofluorescent histochemical staining}

After deep anesthesia was induced using pentobarbital (60 $\mathrm{mg} \mathrm{kg}^{-1}$, i.p.), rats were perfused through the ascending aorta with $100 \mathrm{ml} 0.9 \%$ saline followed by $500 \mathrm{ml} 0.1 \mathrm{M}$ phosphate buffer (PB, pH 7.3) that contained $4 \%$ paraformaldehyde and $2 \%$ picric acid. After perfusion, the L5 spinal segment was removed and postfixed in the same fixative for 2-4 $\mathrm{h}$ and then cryoprotected for $24 \mathrm{~h}$ at $4^{\circ} \mathrm{C}$ in $0.1 \mathrm{M} \mathrm{PB}$ that contained $30 \%$ sucrose. Transverse frozen spinal sections $(30 \mu \mathrm{m}$ in thickness) were cut with a cryostat (Leica CM1800; Heidelberg, Germany) and collected serially into three dishes. Each dish contained a complete set of serial sections.

The sections in the first dish were rinsed in $0.01 \mathrm{M}$ phosphate-buffered saline (PBS, pH 7.3) three times (10 min each), blocked with $2 \%$ goat serum in $0.01 \mathrm{M} \mathrm{PBS}$ that contained $0.3 \%$ Triton X-100 for $1 \mathrm{~h}$ at room temperature $\left(\mathrm{RT}, 20-25^{\circ} \mathrm{C}\right)$, and then used for immunofluorescent histochemical staining. The sections were incubated overnight at $4^{\circ} \mathrm{C}$ with rabbit anti-phosphorylated JNK (pJNK) antibody (1:1000; Cell Signaling Technology, Beverly, MA, USA). The sections were washed three times in $0.01 \mathrm{M}$ PBS (10 min each) and then incubated for $4 \mathrm{~h}$ at RT with the secondary antibody: Alexa 488 donkey anti-rabbit IgG (1:500; Invitrogen, Carlsbad, CA).

Other primary antibodies used in this study were monoclonal antibodies: mouse anti-neuronal-specific nuclear protein (NeuN) (1:3000; Chemicon, Temecula, CA), mouse anti-glial fibrillary acidic protein (GFAP) (1:5000; Chemicon, Temecula, CA), and mouse anti-cd11b clone ox42 (1:500; Abcam, Cambridge, UK). For double immunofluorescence, sections were incubated with a mixture of two primary antibodies followed by a mixture of the two respective secondary antibodies (Alexa 488 donkey antirabbit IgG and Alexa 594 donkey anti-mouse IgG, 1:500; Invitrogen, Carlsbad, CA). Confocal images were obtained using a confocal laser microscope (FV1000; Olympus, Tokyo, Japan) and digital images were captured with Fluoview 1000 (Olympus). The excitation peak wavelength was $495 \mathrm{~nm}$ for Alexa 488 or $590 \mathrm{~nm}$ for Alexa 594, and the emission peak wavelength was $519 \mathrm{~nm}$ for Alexa 488 or $617 \mathrm{~nm}$ for Alexa 594.

The specificity of the staining was tested on the sections in the second dish by omission of the primary specific antibodies. No immunoreactive products were found on these sections. Sections in the third dish were used for Nissl staining (data not shown).

\section{Western blot}

Animals were sacrificed after inducing deep anesthesia (pentobarbital, $60 \mathrm{mg} \mathrm{kg}^{-1}$, i.p.) and the L5 dorsal horns 
were quickly removed. The spinal dorsal horn was then dissected using the "open book" method [10]. Briefly, the L5 spinal cord segment was dissected according to the termination of the L4 and L5 dorsal roots. Then, the spinal segment was cut into a left and right half from the midline. Finally, the left half was further split into the dorsal and ventral horns at the level of the central canal. The selected region was homogenized with a hand-held pestle in sodium dodecyl sulfate (SDS) sample buffer (10 ml mg ${ }^{-1}$ tissue), which contained a cocktail of proteinase and phosphatase inhibitors. The electrophoresis samples were heated at $100^{\circ} \mathrm{C}$ for $5 \mathrm{~min}$ and loaded onto $10 \%$ SDS-polyacrylamide gels with standard Laemmli solutions (Bio-Rad Laboratories, CA, USA). The proteins were electroblotted onto a polyvinylidene difluoride membrane (PVDF, Immobilon-P, Millipore, Billerica, MA, USA). The membranes were placed in a blocking solution, which contained Tris-buffered saline with $0.02 \%$ Tween (TBS-T) and 5\% non-fat dry milk, for $1 \mathrm{~h}$, and incubated overnight under gentle agitation with primary antibody rabbit anti-pJNK (1:1000; Cell Signaling Technology, Beverly, MA), rabbit anti-JNK (1:1000; Cell Signaling Technology) and mouse anti- $\beta$-actin (1:1000; Sigma, St Louis, MO, USA) respectively. Bound primary antibodies were detected with a horseradish peroxidase (HRP)-conjugated anti-rabbit or anti-mouse secondary antibody (1:10000; Amersham Pharmacia Biotech Inc., Piscataway, NJ, USA). Between each step, the immunoblots were rinsed with TBS-T. All reactions were detected by the enhanced chemiluminescence (ECL) detection method (Amersham). The densities of protein blots were analyzed by using Labworks Software (Ultra-Violet Products, UK). The densities of pJNK, JNK and $\beta$-actin immunoreactive bands were quantified with background subtraction. Squares of identical sizes were drawn around each band to measure density, and background near that band was subtracted. Since $\beta$-actin levels didn't change significantly after inflammation and nerve injury [27], we used $\beta$-actin levels as loading controls, and pJNK or JNK levels were normalized against $\beta$-actin levels.

\section{Quantification and statistical analysis}

All data were collected and analyzed by researchers blinded to the surgery and reagents used.

Data from immunofluorescence were calculated as detailed in our previous report [28]. For quantification of pJNK-immunopositive cell profiles in spinal cord, five nonadjacent sections $(30 \mu \mathrm{m})$ from L5 segments were selected randomly from each animal. In each group, 6 rats were used for statistical analysis. Images $(450 \times 338$ $\mu \mathrm{m}^{2}$ ) of the medial two-thirds of the superficial dorsal horn (laminas I-III) were captured under a $20 \times$ objective [8]. All positively stained cells in the area were evaluated using a computer-assisted image analysis program (MetaMorph 6.1), which set low and high thresholds for immunofluorescent intensity determined by the signal. The same configuration was used to measure cell areas in all experimental groups. The measured areas were transferred to Excel automatically for the following statistic analysis. MetaMorph 6.1 was calibrated to provide standardization of area measurements. A standardized field area was sampled arbitrarily from regions within randomly selected dorsal horn sections [8]. Data from immunofluorescence were expressed as fold change against that of the Sham-Saline (or Sham) group. ANOVA followed by the least significant difference test was used for statistical analysis.

Data from western blots are expressed as mean \pm SD . Differences in changes of values over time of each group were tested using one-way ANOVA, followed by the least significant difference test. Data from the vonFrey test are presented as mean \pm SD and were analyzed as the interpolated 50\% threshold (absolute threshold) in $\log$ base 10 of stimulus intensity (monofilament stiffness in milligrams $\times 10$ ). Repeated measures ANOVA (with Bonferroni confidence interval adjustment) was used and conducted for analysis. Data from the rotarod test are presented as mean $\pm S D$, and repeated measures ANOVA (with Bonferroni confidence interval adjustment) was also used. All statistical analyses were performed using SPSS $^{\circledR}$ version 16.0 software (SPSS Inc., Chicago, IL, USA). $P<0.05$ was considered statistically significant.

\section{Results}

SNL induces significant ipsilateral up-regulation of astrocytic pJNK in spinal dorsal horn

SNL induced a marked pJNK up-regulation in ipsilateral spinal cord (Figure 1A), especially in the superficial dorsal horn, while few pJNK-immunopositive cells could be detected in contralateral spinal dorsal horn 10 days after SNL (Figure 1B). There was no significant difference in pJNK expression in spinal dorsal horn between ShamSaline (Figure 1C) and naïve (Figure 1D) groups. However, compared with that of the Sham-Saline group, SNL induced a much higher expression of pJNK in the spinal dorsal horn (Figure 1E), indicated by increased density of immunoreactive staining in high-magnification images (Figure 1F).

In order to detect the cellular localization of pJNK expression, double immunofluorescent staining with antibodies against pJNK and the neuronal marker NeuN, the microglial specific marker OX42, or the astrocytic specific marker GFAP was performed, respectively. No colocalization could be observed either between pJNK and NeuN (Figure 2: A-A"), or between pJNK and OX42 (Figure 2: B-B"), which suggests that neither neurons nor 


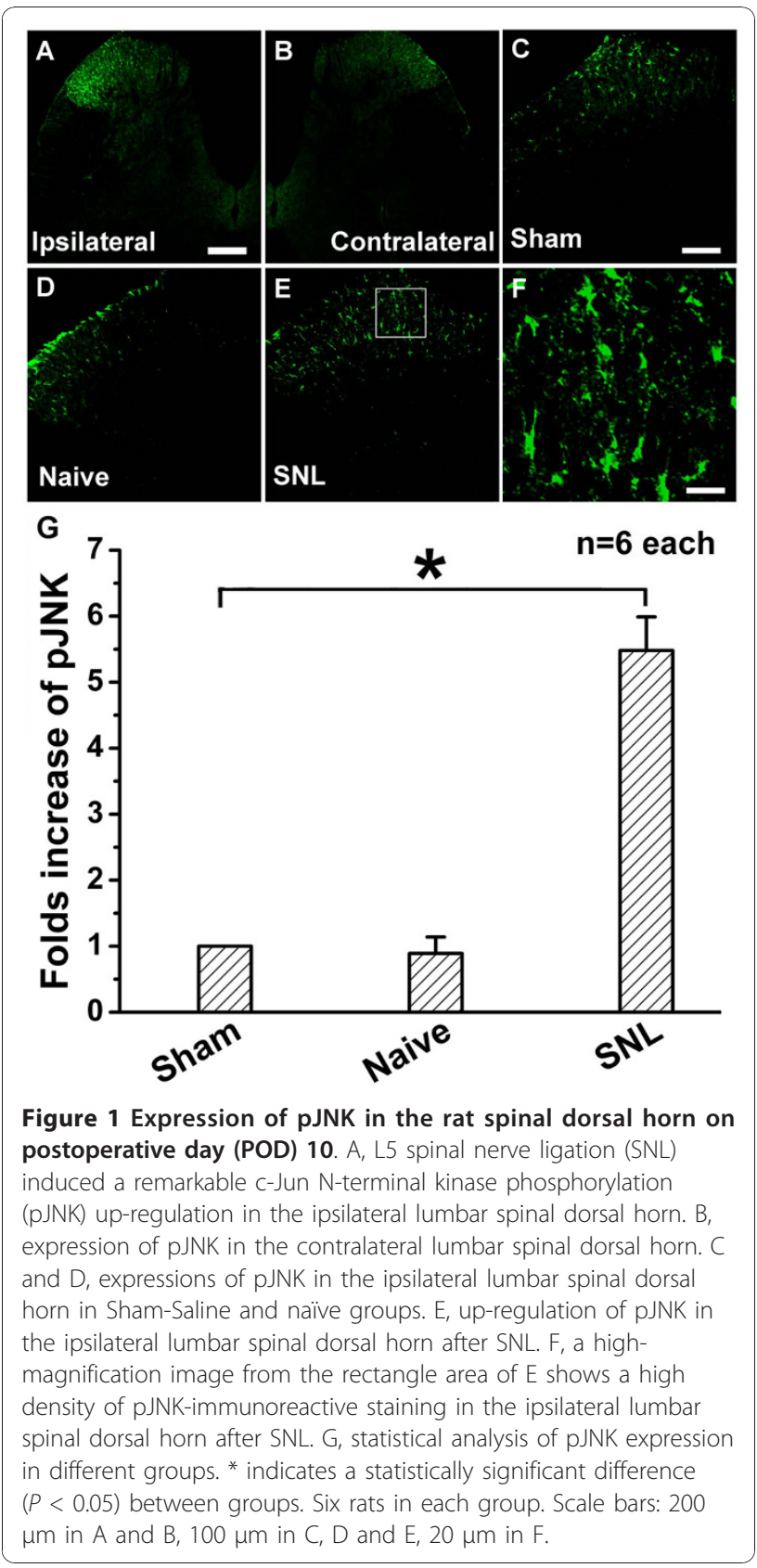

microglia express pJNK in spinal dorsal horn 10 days after SNL. However, all pJNK-positive cells were GFAPpositive astrocytes (Figure 2: C-C").

\section{Effects of ketamine on motor functions indicated by rotarod testing}

Nociceptive behavioral results could be easily influenced as a result of motor dysfunction. In order to assess whether the dosages of ketamine used in the present study (30, 100 and $300 \mu \mathrm{g} \mathrm{kg}^{-1}$ ) could produce impairment of motor functions, 18 otherwise experiment-free rats were assessed using a rotarod test. Intrathecal

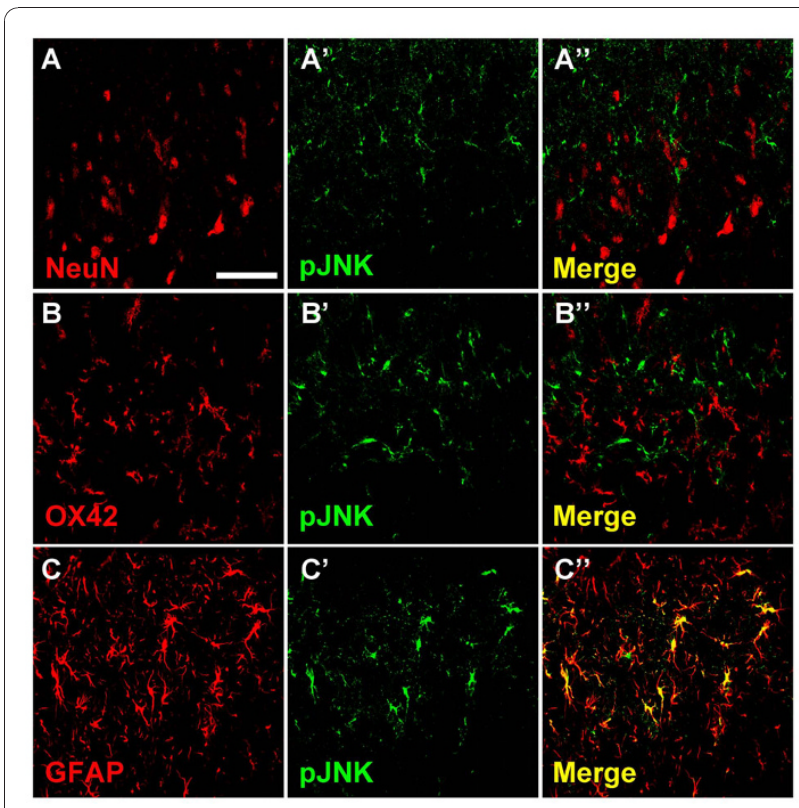

Figure 2 Cellular localization of pJNK expression in spinal dorsal horn after SNL. High-magnification images demonstrated the results of double immunofluorescent histochemical staining with NeuN and pJNK $\left(A-A^{\prime \prime}\right)$, with OX-42 and pJNK (B-B"), or with glial fibrillary acidic protein (GFAP) and pJNK (C-C'). Scale bar: $50 \mu \mathrm{m}$.

ketamine $\left(30,100\right.$ and $\left.300 \mu \mathrm{g} \mathrm{kg}^{-1}\right)$ did not affect the motor performance of rats $30 \mathrm{~min}$ after injection compared with their own baseline (Figure 3). Furthermore, repeated administrations did not influence motor function either.

Ketamine attenuates SNL-induced mechanical allodynia in a dose-dependent manner

In order to verify the effects of intrathecal ketamine on SNL-induced neuropathic pain, ketamine was injected once a day with three different dosages and changes in PWT were observed 30 min after injection, from POD 8 to POD 10 (Figure 4).

SNL induced significant mechanical allodynia as shown in the SNL-Saline group. Compared with that of the SNL-Saline group, intrathecal injection of $30 \mu \mathrm{g} \mathrm{kg}^{-1}$ ketamine did not influence PWT (Figure 4). Intrathecal ketamine $\left(100 \mu \mathrm{g} \mathrm{kg}^{-1}\right)$ elevated PWT significantly after administration $(P<0.05$, compared with that of SNLSaline). Furthermore, a higher dose of ketamine (300 $\mu \mathrm{g}$ $\left.\mathrm{kg}^{-1}\right)$ apparently elevated PWT $(P<0.05$, compared with that of SNL-ketamine $100 \mu \mathrm{g} \mathrm{kg}^{-1}$ ) (Figure 4). However, neither high $\left(300 \mu \mathrm{g} \mathrm{kg}^{-1}\right)$ nor lower doses (30 and 100 $\mu \mathrm{g} \mathrm{kg}{ }^{-1}$, data not shown) of ketamine changed the basal threshold in the sham-operated group (Figure 4). These results demonstrate that intrathecal ketamine $(30,100$ and $300 \mu \mathrm{g} \mathrm{kg}^{-1}$ ) produces an effective and reliable 


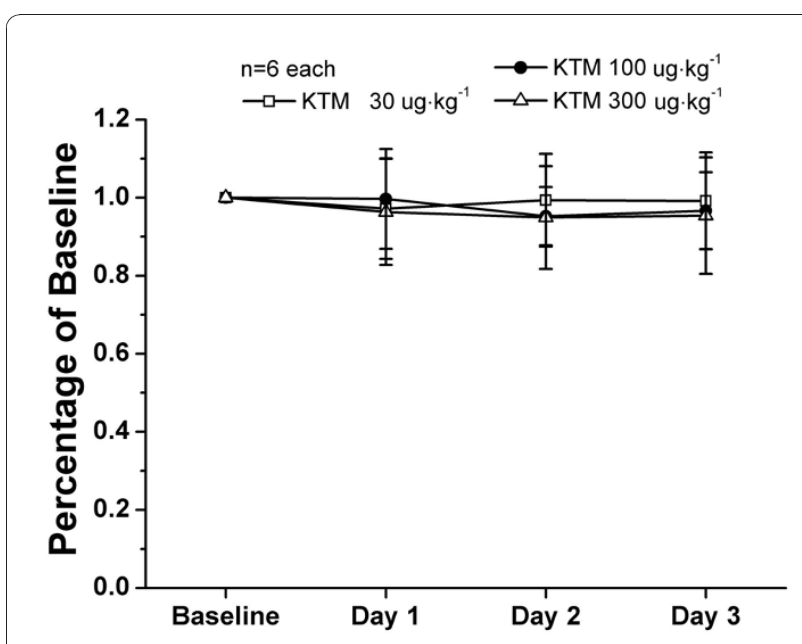

Figure 3 Effects of ketamine on motor performance of rats in the rotarod test. After recording baseline response, ketamine (30, 100 or $300 \mathrm{mg} \mathrm{kg}^{-1}$ ) was administered intrathecally and rotarod test was performed 30 min later once a day for 3 days, respectively. Compared with the baseline response, no statistical differences were obtained from rotarod test after intrathecal ketamine. There were six rats in each group. KTM: ketamine.

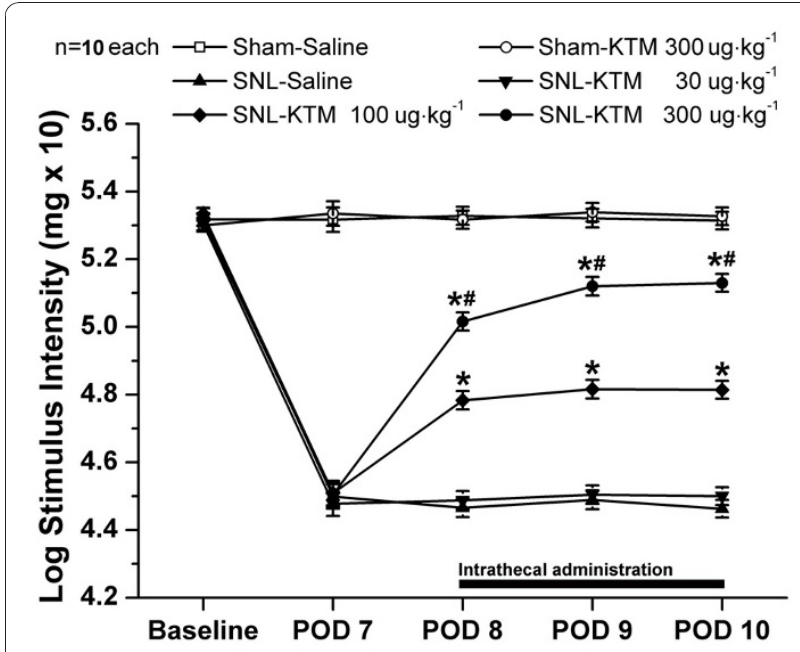

Figure 4 Effects of intrathecal ketamine on SNL-induced mechanical allodynia. SNL induced significant mechanical allodynia as shown by von-Frey tests. Intrathecal $300 \mathrm{mg} \mathrm{kg}^{-1}$ ketamine did not change the normal pain threshold of the shamoperated group. Intrathecal ketamine $\left(100\right.$ and $\left.300 \mu \mathrm{g} \mathrm{kg}^{-1}\right)$ showed an effective and reliable anti-allodynia effect in a dose-dependent manner on SNL-induced neuropathic pain, whereas, intrathecally $30 \mathrm{mg} \mathrm{kg}^{-1}$ of ketamine did not influence pain threshold after SNL at all. Drugs were given intrathecally once a day from POD 8 to POD 10. ${ }^{*} P<0.05$, compared with that of SNL-Saline group. ${ }^{*} P<0.05$, compared with that of SNL-ketamine $100 \mu \mathrm{g} \mathrm{kg}^{-1}$ group. There were ten rats in each group. KTM: ketamine. anti-allodynia effect in a dose-dependent manner on SNL-induced neuropathic pain.

\section{Effects of intrathecal ketamine on SNL-induced pJNK expression}

Next, the effect of intrathecal ketamine on JNK phosphorylation was tested. Intrathecal ketamine (300 $\mu \mathrm{g} \mathrm{kg}^{-1}$ ) did not show any effect on pJNK expression in sham operated rats compared with that of the ShamSaline group (Figure 5:A, B), which was also verified by western blot (Figure 6A). SNL enhanced expression of pJNK in spinal dorsal horn (Figure $5 \mathrm{C}$ ), which was

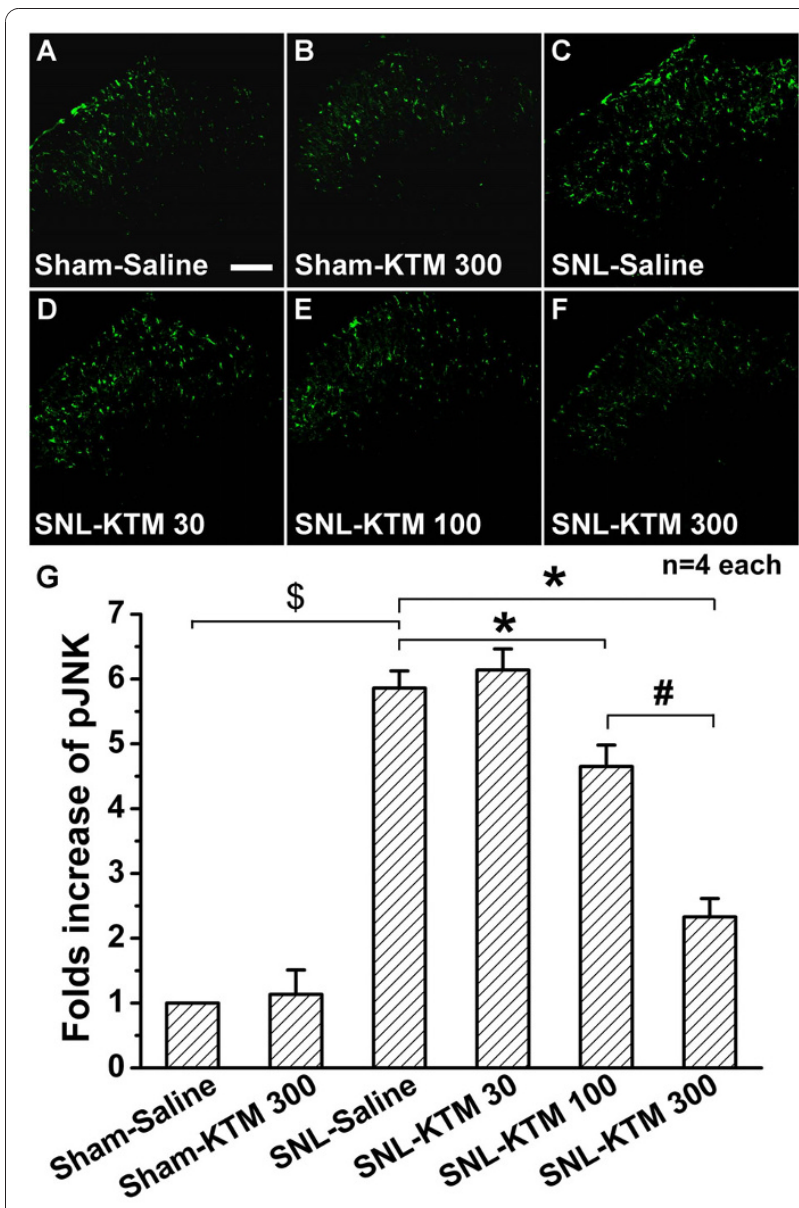

Figure 5 Effect of intrathecal ketamine on SNL-induced pJNK expression. Only a little pJNK expression could be detected in the Sham-Saline group (A), and intrathecal ketamine $\left(300 \mu \mathrm{g} \mathrm{kg}^{-1}\right)$ had no effect on pJNK expression in sham-operated rats (B). SNL induced significant pJNK expression (C), which could be suppressed by intrathecal ketamine in a dose-dependent manner (D-F). G, statistical analysis of the pJNK expressions after different treatments. *, \# or $\$$ each indicates statistically significant difference with $P<$ 0.05 between groups. Four rats in each group. Scale bar: $100 \mu \mathrm{m}$. KTM: ketamine. 

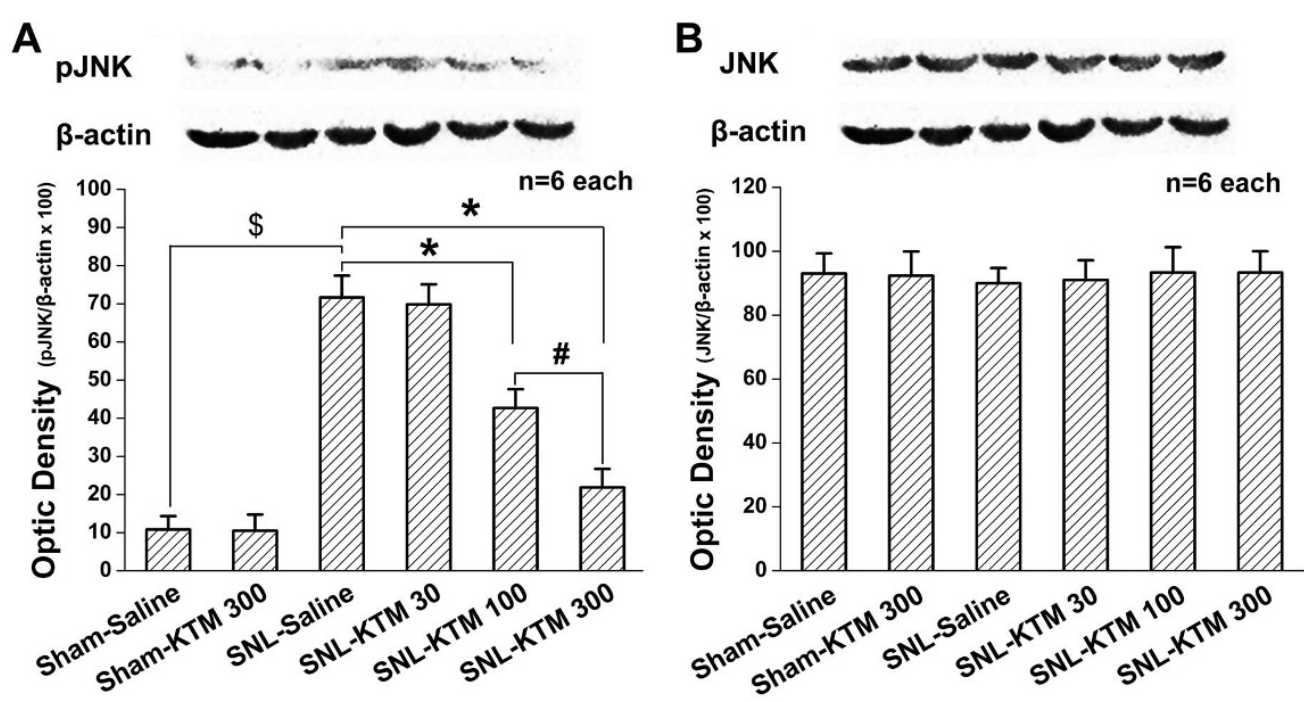

Figure 6 Western blot quantification of JNK and pJNK expressions after different treatments. A: Compared with that of Sham-Saline group, no difference could be detected in pJNK expression after intrathecal ketamine $\left(300 \mathrm{\mu g} \mathrm{kg}^{-1}\right)$ to sham-operated rats, whereas SNL apparently induced pJNK up-regulation. However, intrathecal ketamine down-regulated SNL-induced pJNK expression in a dose-dependent manner, which was in accord with the immunofluorescent histochemical staining result. B: Spinal JNK protein expression did not show any obvious difference after different treatments. ${ }^{*}, \#$ or $\$$ each indicates statistically significant difference with $P<0.05$ between groups. There were six rats in each group. KTM: ketamine.

increased about 5.62-fold in the SNL-Saline group compared with that of the Sham-Saline group (Figure 6A, $p<$ 0.05). Intrathecal ketamine produced a dose-dependent effect on suppression of SNL-induced pJNK up-regulation in spinal dorsal horn (Figure 5:D-F, Figure 6A). Intrathecal $30 \mu \mathrm{g} \mathrm{kg}^{-1}$ ketamine did not show any obvious effect on pJNK expression compared with that of the SNL-Saline group (Figure 5D, Figure 6A). However, the expression of pJNK was significantly attenuated to $59.54 \%$ of the SNL-Saline group by intrathecal $100 \mu \mathrm{g} \mathrm{kg}$ ${ }^{1}$ of ketamine (Figure 5E, Figure 6A, $P<0.05$ ). Moreover, intrathecal $300 \mu \mathrm{g} \mathrm{kg}^{-1}$ ketamine significantly down-regulated pJNK expression (Figure 5F), to just $30.46 \%$ that of the SNL-Saline group (Figure 6A, $P<0.05$ ). Furthermore, intrathecal $300 \mu \mathrm{g} \mathrm{kg}^{-1}$ ketamine showed stronger effects on pJNK expression compared with that of the SNL-ketamine $100 \mu \mathrm{g} \mathrm{kg}^{-1}$ ketamine group (Figure 6A, $P<0.05$ ). These results suggest that intrathecal ketamine can effectively inhibit pJNK up-regulation in a dose-dependent manner. However, neither SNL operation nor intrathecal ketamine $\left(30,100\right.$ and $\left.300 \mu \mathrm{g} \mathrm{kg}^{-1}\right)$ had any obvious effect on spinal JNK expression (Figure 6B). This result suggests that intrathecal ketamine only suppresses activation of the JNK pathway, without changing spinal expression of total JNK.

\section{Discussion}

We have previously shown that intrathecal ketamine attenuates SNL-induced neuropathic pain and inhibits astrocytic activation in lumbar spinal dorsal horn [21]. However, the molecular mechanisms of how ketamine influences astrocytic function are unclear. In the present study we show that intrathecal ketamine significantly suppresses nerve injury-induced mechanical allodynia. More interestingly, the activation of JNK, which is selectively expressed in spinal astrocytes, could be inhibited by ketamine. Our results suggest that inhibiting activation of astrocytic JNK may play an important role in the analgesic effect of ketamine.

JNK is a member of the mitogen-activated protein kinase (MAPK) family, which has been shown to have a critical role in intracellular signal transduction. It has been confirmed that both JNK1 and JNK2 are constitutively expressed in the spinal cord; however, only phosphorylated JNK1 (pJNK1, active form) is increased after SNL [8]. Thus, for the present study, we focused on the phosphorylated form of JNK (pJNK1) after SNL and drug administration. Present results showed that JNK phosphorylation was highly up-regulated and specifically localized in spinal astrocytes. These results are in accord with a previous report that SNL induces a slow ( $>3 \mathrm{~d}$ ) and persistent ( $>21 \mathrm{~d}$ ) activation of JNK in spinal astrocytes [8].

Previous reports have confirmed that the JNK pathway is preferentially activated in spinal astrocytes $[8,11,29,30]$, which is different from the pERK and p-p38 pathways at the spinal level after nerve injury $[10,15]$. It also has been validated that intrathecal administration of a JNK inhibitor can suppress spinal 
astrocytic activation and relieve neuropathic pain after SNL $[8,31]$. Present study showed that ketamine suppressed pJNK expression in a dose-dependent manner. Therefore, this may be the underlying molecular mechanism for ketamine to inhibit astrocytic activation and to relief mechanical allodynia, especially after nerve injury.

Ketamine has been reported to exert anti-inflammatory effects on macrophages stimulated with lipopolysaccharide (LPS) in vitro and in vivo, with involvement of toll-like receptors (TLRs) and MAPK pathways (JNK and ERK) $[23,32]$. Glial cells, the "immunocytes" of the CNS, somewhat like macrophages, have been confirmed to be involved in the immune response in neuropathic pain condition [33]. Furthermore, it has been reported that ketamine reduces LPS-induced tumor necrosis factor (TNF) $-\alpha$, and prostaglandin E2 (PGE2) production in astrocyte cultures to inhibit some LPS-induced astrocytic inflammatory responses [22]. Therefore, intrathecal ketamine could attenuate SNL-induced mechanical allodynia by suppression of astrocytic JNK activation, which has been verified by the present research. However, how does ketamine function on astrocytic JNK activation after intrathecal injection?

Ketamine has been recognized as a nonselective NMDA receptors antagonist. A previous study has shown that astrocytic activation is markedly suppressed by treatment with the NR2B- (a subunit of NMDA receptor) selective antagonist ifenprodil in neuron-glia co-cultures, but not in purified astrocytes [34]. For this reason, blocking NMDA receptor on neurons might be an indirect method to suppress astrocytic activation with pJNK expression after intrathecal ketamine.

Evidence indicates that proinflammatory cytokines such as TNF- $\alpha$, interleukin (IL)-1 $\beta$, and IL- 6 are primary activators of JNK pathway $[12,35,36]$. Activation of JNK pathways also can lead to production and release of multiple proinflammatory mediators [17,37], which form an excitatory feedback loop among glial cells and neurons resulting in enhanced excitability and plasticity $[7,33,38,39]$. Ketamine can exert anti-inflammatory effects in vitro and in vivo [40-43]. It is reported that ketamine can inhibit the effects of TNF- $\alpha$ on cellular signal transmission [43]. Moreover, it is also confirmed that ketamine can reduce astrocytic TNF- $\alpha$ expression and release [22], which may break down the positive feedback loop and alleviate the neuropathic pain. Consequently, suppression of proinflammatory factors expression after peripheral nerve injury could be an option for down-regulating pJNK expression by intrathecal ketamine.

In addition, accumulating studies have indicated that TLRs, expressed widely on spinal glia, are involved in the development of neuropathic pain after nerve injury [44-46]. It has been suggested that glial TLRs could be new targets for treatment of neuropathic pain [45]. TLRs in spinal cord may have a role in the early establishment of neuropathic pain after peripheral nerve injury $[44,46]$. A previous report indicated that TLR3 could mediate signals that induce proinflammatory cytokine and chemokine gene expression in astrocytes, which could be prevented by SP600125, a pharmacological inhibitor of JNK [37]. This report indicates that activation of JNK is required for TLR3-mediated proinflammatory cytokine and chemokine gene expression in astrocytes. Additionally, ketamine can inhibit proinflammatory gene expression, such as TNF- $\alpha$, IL-1 $\beta$, IL-6 and so on, possibly by suppressing TLRs-mediated signaltransduction [22,32,40,47]. Accordingly, ketamine might act on TLRs located on astrocytes and thus inhibit astrocytic JNK activation and, consequently, neuropathic pain.

\section{Conclusions}

Given the important role of the astrocytic JNK pathway in neuropathic pain, inhibiting JNK activation could be useful in the treatment of neuropathic pain. The present study shows that intrathecal ketamine can down-regulate astrocytic pJNK expression and attenuate mechanical allodynia in a dose-dependent manner. Although more detailed mechanisms need to be further investigated and classified, the present work provides a potential strategy for treating neuropathic pain.

\section{Acknowledgements}

This work was supported by the grants from the National Natural Science Foundation of China (30901400, 30772073, 30771133 and 30971123), Eleventh Five-Year Project of Science and Technology (06G093) and National Program of Basic Research of China (G2006CB500808). Our great thanks also go for the critical scientific opinions and help in English by Dr. Jerome Staal from the Menzies Research Institute Tasmania in Australia.

\section{Author details}

${ }^{1}$ Department of Anesthesiology, School of Stomatology, Fourth Military Medical University, Xi'an, 710032, PR China. ${ }^{2}$ Department of Anatomy, Histology and Embryology, K. K. Leung Brain Research Centre, Fourth Military Medical University, Xi'an, 710032, PR China.

\section{Authors' contributions}

XPM and $\mathrm{HZ}$ performed the animal surgery, carried out the Western blot study and drafted the manuscript. MZZ and YYW carried out the immunofluorescence and western blot. WW (Wei Wang) performed the behavioral test. WW (Wen Wang) participated in producing graphics and performed the statistical analysis. LXX and YQL conceived the study, and participated in its design and coordination. All authors read and approved the final manuscript.

\section{Competing interests}

The authors declare that they have no competing interests.

Received: 27 October 2010 Accepted: 24 January 2011 Published: 24 January 2011 


\section{References}

1. Watkins LR, Martin D, Ulrich P, Tracey KJ, Maier SF: Evidence for the involvement of spinal cord glia in subcutaneous formalin induced hyperalgesia in the rat. Pain 1997, 71:225-235

2. Meunier A, Mauborgne A, Masson J, Mallet J, Pohl M: Lentiviral-mediated targeted transgene expression in dorsal spinal cord glia: tool for the study of glial cell implication in mechanisms underlying chronic pain development. J Neurosci Methods 2008, 167:148-159.

3. Garry EM, Delaney A, Blackburn-Munro G, Dickinson T, Moss A, Nakalembe I, Robertson DC, Rosie R, Robberecht P, Mitchell R, Fleetwood-Walker SM: Activation of p38 and p42/44 MAP kinase in neuropathic pain: involvement of VPAC2 and NK2 receptors and mediation by spinal glia. Mol Cell Neurosci 2005, 30:523-537.

4. Milligan ED, Twining C, Chacur M, Biedenkapp J, O'Connor K, Poole S, Tracey K, Martin D, Maier SF, Watkins LR: Spinal glia and proinflammatory cytokines mediate mirror-image neuropathic pain in rats. J Neurosci 2003, 23:1026-1040

5. Watkins LR, Milligan ED, Maier SF: Spinal cord glia: new players in pain. Pain 2001, 93:201-205.

6. Tsuda M, Inoue K, Salter MW: Neuropathic pain and spinal microglia: a big problem from molecules in "small" glia. Trends Neurosci 2005, 28:101-107.

7. Scholz J, Woolf CJ: The neuropathic pain triad: neurons, immune cells and glia. Nat Neurosci 2007, 10:1361-1368.

8. Zhuang ZY, Wen YR, Zhang DR, Borsello T, Bonny C, Strichartz GR, Decosterd I, Ji RR: A peptide c-Jun N-terminal kinase (JNK) inhibitor blocks mechanical allodynia after spinal nerve ligation: respective roles of JNK activation in primary sensory neurons and spinal astrocytes for neuropathic pain development and maintenance. J Neurosci 2006, 26:3551-3560.

9. Raghavendra V, Tanga F, DeLeo JA: Inhibition of microglial activation attenuates the development but not existing hypersensitivity in a rat model of neuropathy. J Pharmacol Exp Ther 2003, 306:624-630.

10. Zhuang ZY, Gerner P, Woolf CJ, Ji RR: ERK is sequentially activated in neurons, microglia, and astrocytes by spinal nerve ligation and contributes to mechanical allodynia in this neuropathic pain model. Pain 2005, 114:149-159.

11. Ji RR, Gereau RWt, Malcangio M, Strichartz GR: MAP kinase and pain. Brain Res Rev 2009, 60:135-148

12. Gao YJ, Ji RR: Activation of JNK pathway in persistent pain. Neurosci Lett 2008, 437:180-183.

13. Xu M, Bruchas MR, Ippolito DL, Gendron L, Chavkin C: Sciatic nerve ligation-induced proliferation of spinal cord astrocytes is mediated by kappa opioid activation of p38 mitogen-activated protein kinase. $J$ Neurosci 2007, 27:2570-2581.

14. Piao ZG, Cho IH, Park CK, Hong JP, Choi SY, Lee SJ, Lee S, Park K, Kim JS, Oh SB: Activation of glia and microglial p38 MAPK in medullary dorsal horn contributes to tactile hypersensitivity following trigeminal sensory nerve injury. Pain 2006, 121:219-231.

15. Jin SX, Zhuang ZY, Woolf CJ, Ji RR: p38 mitogen-activated protein kinase is activated after a spinal nerve ligation in spinal cord microglia and dorsal root ganglion neurons and contributes to the generation of neuropathic pain. J Neurosci 2003, 23:4017-4022

16. Tsuda M, Mizokoshi A, Shigemoto-Mogami Y, Koizumi S, Inoue K: Activation of p38 mitogen-activated protein kinase in spinal hyperactive microglia contributes to pain hypersensitivity following peripheral nerve injury. Glia 2004, 45:89-95.

17. Gao YJ, Zhang L, Samad OA, Suter MR, Yasuhiko K, Xu ZZ, Park JY, Lind AL, $M a$ Q, Ji RR: JNK-induced MCP-1 production in spinal cord astrocytes contributes to central sensitization and neuropathic pain. J Neurosci 2009, 29:4096-4108.

18. Nadeson R, Tucker A, Bajunaki E, Goodchild CS: Potentiation by ketamine of fentanyl antinociception. I. An experimental study in rats showing that ketamine administered by non-spinal routes targets spinal cord antinociceptive systems. Br J Anaesth 2002, 88:685-691.

19. Christoph T, Schiene K, Englberger W, Parsons CG, Chizh BA: The antiallodynic effect of NMDA antagonists in neuropathic pain outlasts the duration of the in vivo NMDA antagonism. Neuropharmacology 2006, 51:12-17.

20. Kiefer RT, Rohr P, Ploppa A, Dieterich HJ, Grothusen J, Koffler S, Altemeyer KH, Unertl K, Schwartzman RJ: Efficacy of Ketamine in
Anesthetic Dosage for the Treatment of Refractory Complex Regional Pain Syndrome: An Open-Label Phase II Study. Pain Med 2008, 9:1173-1201.

21. Mei X, Wang W, Wang W, Li Y, Zhang H, Wu S, Li Y, Xu L: Inhibiting astrocytic activation: a novel analgesic mechanism of ketamine at the spinal level? J Neurochem 2009, 109:1691-1700.

22. Shibakawa YS, Sasaki Y, Goshima Y, Echigo N, Kamiya Y, Kurahashi K, Yamada $Y$, Andoh T: Effects of ketamine and propofol on inflammatory responses of primary glial cell cultures stimulated with lipopolysaccharide. Br J Anaesth 2005, 95:803-810.

23. Wu GJ, Chen TL, Ueng YF, Chen RM: Ketamine inhibits tumor necrosis factor-alpha and interleukin-6 gene expressions in lipopolysaccharide-stimulated macrophages through suppression of toll-like receptor 4-mediated c-Jun N-terminal kinase phosphorylation and activator protein-1 activation. Toxicol Appl Pharmacol 2008, 228:105-113.

24. Zimmermann M: Ethical guidelines for investigations of experimental pain in conscious animals. Pain 1983, 16:109-110.

25. Kim SH, Chung JM: An experimental model for peripheral neuropathy produced by segmental spinal nerve ligation in the rat. Pain 1992. 50:355-363.

26. Chaplan SR, Bach FW, Pogrel JW, Chung JM, Yaksh TL: Quantitative assessment of tactile allodynia in the rat paw. J Neurosci Methods 1994 53:55-63.

27. Guo W, Wang H, Watanabe M, Shimizu K, Zou S, LaGraize SC, Wei F, Dubner R, Ren K: Glial-cytokine-neuronal interactions underlying the mechanisms of persistent pain. J Neurosci 2007, 27:6006-6018.

28. Wang W, Wang W, Mei X, Huang J, Wei Y, Wang Y, Wu S, Li Y: Crosstalk between spinal astrocytes and neurons in nerve injury-induced neuropathic pain. PLoS One 2009, 4:e6973.

29. Ma W, Quirion R: Partial sciatic nerve ligation induces increase in the phosphorylation of extracellular signal-regulated kinase (ERK) and c-Jun $\mathrm{N}$-terminal kinase (JNK) in astrocytes in the lumbar spinal dorsal horn and the gracile nucleus. Pain 2002, 99:175-184.

30. Katsura H, Obata K, Miyoshi K, Kondo T, Yamanaka H, Kobayashi K, Dai Y, Fukuoka T, Sakagami M, Noguchi K: Transforming growth factor-activated kinase 1 induced in spinal astrocytes contributes to mechanical hypersensitivity after nerve injury. Glia 2008, 56:723-733.

31. Obata K, Yamanaka H, Kobayashi K, Dai Y, Mizushima T, Katsura H, Fukuoka T, Tokunaga A, Noguchi K: Role of mitogen-activated protein kinase activation in injured and intact primary afferent neurons for mechanical and heat hypersensitivity after spinal nerve ligation. $J$ Neurosci 2004, 24:10211-10222.

32. Chang HC, Lin KH, Tai YT, Chen JT, Chen RM: Lipoteichoic acid-induced TNF-alpha and IL- 6 gene expressions and oxidative stress production in macrophages are suppressed by ketamine through downregulating Tolllike receptor 2-mediated activation oF ERK1/2 aND NFkappaB. Shock 2010, 33:485-492.

33. Milligan ED, Watkins LR: Pathological and protective roles of glia in chronic pain. Nat Rev Neurosci 2009, 10:23-36.

34. Kato $H$, Narita M, Miyatake M, Yajima Y, Suzuki T: Role of neuronal NR2B subunit-containing NMDA receptor-mediated $\mathrm{Ca} 2+$ influx and astrocytic activation in cultured mouse cortical neurons and astrocytes. Synapse 2006, 59:10-17.

35. Wu CY, Hsieh HL, Sun CC, Yang CM: IL-1 beta induces MMP-9 expression via a Ca2+-dependent CaMKII/JNK/c-JUN cascade in rat brain astrocytes. Glia 2009, 57:1775-1789.

36. Wang X, Fu S, Wang Y, Yu P, Hu J, Gu W, Xu XM, Lu P: Interleukin-1beta mediates proliferation and differentiation of multipotent neural precursor cells through the activation of SAPK/JNK pathway. Mol Cell Neurosci 2007, 36:343-354.

37. Park C, Lee S, Cho IH, Lee HK, Kim D, Choi SY, Oh SB, Park K, Kim JS, Lee SJ: TLR3-mediated signal induces proinflammatory cytokine and chemokine gene expression in astrocytes: differential signaling mechanisms of TLR3-induced IP-10 and IL-8 gene expression. Glia 2006, 53:248-256.

38. Lee KM, Jeon SM, Cho HJ: Tumor necrosis factor receptor 1 induces interleukin- 6 upregulation through NF-kappaB in a rat neuropathic pain model. Eur J Pain 2009, 13:794-806.

39. Blanco AM, Perez-Arago A, Fernandez-Lizarbe S, Guerri C: Ethanol mimics ligand-mediated activation and endocytosis of IL-1RI/TLR4 receptors via lipid rafts caveolae in astroglial cells. J Neurochem 2008, 106:625-639. 
40. Chen TL, Chang CC, Lin YL, Ueng YF, Chen RM: Signal-transducing mechanisms of ketamine-caused inhibition of interleukin-1 beta gene expression in lipopolysaccharide-stimulated murine macrophage-like Raw 264.7 cells. Toxicol Appl Pharmacol 2009, 240:15-25.

41. Mazar J, Rogachev B, Shaked G, Ziv NY, Czeiger D, Chaimovitz C, Zlotnik M, Mukmenev I, Byk G, Douvdevani A: Involvement of adenosine in the antiinflammatory action of ketamine. Anesthesiology 2005, 102:1174-1181.

42. Shaked G, Czeiger D, Dukhno O, Levy I, Artru AA, Shapira Y, Douvdevani A: Ketamine improves survival and suppresses IL-6 and TNFalpha production in a model of Gram-negative bacterial sepsis in rats. Resuscitation 2004, 62:237-242.

43. Hill GE, Anderson JL, Lyden ER: Ketamine inhibits the proinflammatory cytokine-induced reduction of cardiac intracellular cAMP accumulation. Anesth Analg 1998, 87:1015-1019.

44. Cao L, Tanga FY, Deleo JA: The contributing role of CD14 in toll-like receptor 4 dependent neuropathic pain. Neuroscience 2009, 158:896-903.

45. Bettoni I, Comelli F, Rossini C, Granucci F, Giagnoni G, Peri F, Costa B: Glial TLR4 receptor as new target to treat neuropathic pain: efficacy of a new receptor antagonist in a model of peripheral nerve injury in mice. Glia 2008, 56:1312-1319.

46. Obata K, Katsura H, Miyoshi K, Kondo T, Yamanaka H, Kobayashi K, Dai Y Fukuoka T, Akira S, Noguchi K: Toll-like receptor 3 contributes to spinal glial activation and tactile allodynia after nerve injury. J Neurochem 2008, 105:2249-2259.

47. Yu M, Shao D, Yang J, Feng S, Xu J: Ketamine suppresses intestinal TLR4 expression and NF-kappaB activity in lipopolysaccharide-treated rats. Croat Med J 2006, 47:825-831.

doi:10.1186/1742-2094-8-6

Cite this article as: Mei et al:: Inhibition of spinal astrocytic c-Jun Nterminal kinase (JNK) activation correlates with the analgesic effects of ketamine in neuropathic pain. Journal of Neuroinflammation 2011 8:6.

\section{Submit your next manuscript to BioMed Central and take full advantage of:}

- Convenient online submission

- Thorough peer review

- No space constraints or color figure charges

- Immediate publication on acceptance

- Inclusion in PubMed, CAS, Scopus and Google Scholar

- Research which is freely available for redistribution

Submit your manuscript at www.biomedcentral.com/submit 